\title{
Der Aufschub der Ad-hoc-Publizität nach Art. 17 Abs. 4 MAR zum Schutz der Unternehmensreputation
}

\author{
LARS KLÖHN, Berlin* \\ und \\ Klaus Ulrich SChMOlKe, Erlangen $*$
}

Inhaltsübersicht

ZGR 2016, 866-896

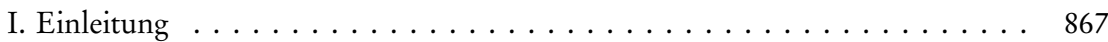

1. Beispielsfälle . . . . . . . . . . . . . . . . . . . 868

2. Bedeutung der Unternehmensreputation in der Praxis . . . . . . . . . . . . . . . 868

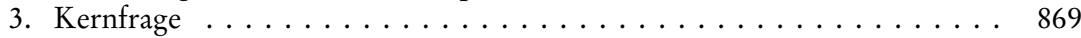

II. Ontologie und Abgrenzung . . . . . . . . . . . . . . . . . . . . . . . . . . 870

1. Handelnder . . . . . . . . . . . . . . . . . . . . . . . 870

2. Rufschädigende Handlungen . . . . . . . . . . . . . . . . . . . . . . . . . 871

3. Fehlende öffentliche Bekanntheit . . . . . . . . . . . . . . . . . . 871

4. Reputationsverlust . . . . . . . . . . . . . . . . . . 871

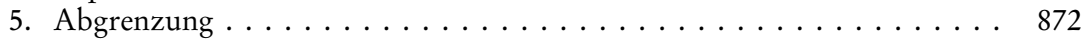

III. Emittenteninteresse . . . . . . . . . . . . . . . . . . . . . . 873

1. Emittenteninteresse als Aktionärsinteresse . . . . . . . . . . . . . . . 874

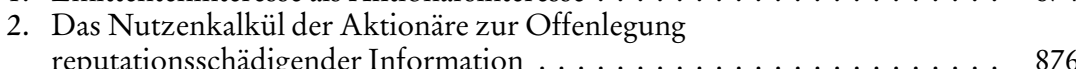

3. Aufschubentscheidung als Prognoseentscheidung des Vorstands . . . . . . 884

4. Entscheidung durch einen befangenen Vorstand . . . . . . . . . . . . 886

IV. Weitere Voraussetzungen von Art. 17 Abs. 4 MAR . . . . . . . . . . . . . . . 892

V. Lösung der Beispielsfälle . . . . . . . . . . . . . . . . . . . . . . . 893

VI. Thesen . . . . . . . . . . . . . . . . . . . . . . . . . 894

Der Vorstand einer börsennotierten AG stößt auf Vorkommnisse in seinem Unternehmen, die dessen Reputation gefäbrden, sobald sie bekannt werden. Die Information ist-auch deshalbkursrelevant. Muss der Vorstand unverzüglich "ad hoc geben"? Oder kann er wegen des drohenden Reputationsverlusts die Veröffentlichung aufschieben? Der vorliegende Beitrag widmet sich dieser Frage. Die Antwort hängt vor allem davon ab, ob ein solcher Aufschub im Emittenteninteresse i.S.d. Art. 17 Abs. 4 MAR läge. Dieses Interesse ist bei rufschädigenden Informationen im Wege einer komplexen Abwägungsentscheidung zu ermitteln, bei der die Vor- und Nachteile des Aufschubs der Ad-hoc-Meldung für die Aktionäre des Emittenten abgeschätzt werden müssen. Der

* Prof. Dr. Lars Klöhn, LL.M. (Harvard) ist Inhaber des Lehrstuhls für Bürgerliches Recht und Wirtschaftsrecht an der Humboldt-Universität zu Berlin.

** Prof. Dr. Klaus Ulrich Schmolke, LL.M. (NYU) ist Inhaber des Lehrstuhls für Bürgerliches Recht, Handels-, Gesellschafts- und Wirtschaftsrecht an der Friedrich-Alexander-Universität Erlangen-Nürnberg. 
Aufsatz macht es sich zur Aufgabe, normative Leitlinien für die gerichtliche Überprüfung dieser Entscheidung zu formulieren. Wie zu zeigen sein wird, bängt deren Inhalt davon ab, ob der konkrete Verdacht besteht, dass die Vorstandsmitglieder, die über den Aufschub der Ad-hocPublizität entscheiden, gesellschaftsrechtliche Verantwortung für den rufschädigenden Vorfall tragen.

Imagine the directors of a listed stock corporation learn of an incident related to the company which has the potential to severely damage the company's reputation as soon as it is revealed to the public. The information about this incident has - not least because of its impact on the company's reputation - a significant price effect in terms of Art. 7(1) MAR. Are the directors required to disclose this piece of information to the public as soon as possible? Or is the board allowed to delay the disclosure because of its adverse effects on the company's reputation? The article at hand addresses these questions. Their answer depends, above all, on whether a delay of the immediate disclosure would accord with the legitimate interests of the issuer as referred to in Art.17(4) MAR. However, to determine what is in the best interest of the issuer when it comes to reputationally sensitive information it takes a complex trade-off decision within which the pros and cons of such a delay for the issuer's shareholders have to be assessed. This article aims at devising normative guidelines for the judicial review of such complex trade-off decisions. As will be shown below, the extent of the judicial review hinges on whether there is a concrete suspicion that the directors' decision about delaying disclosure is not exclusively guided by the shareholders' interests, but (also) by the directors' personal interests or the interests of third parties.

\section{Einleitung}

„It takes 20 years to build a reputation and five minutes to ruin it.“ - dieser Ausspruch Warren Buffetts gehört heute zur Zitaten-Grundausrüstung nicht nur jedes Unternehmensberaters, sondern auch der meisten Wirtschaftsanwälte, Unternehmensjuristen und Compliance-Beauftragten. ${ }^{1}$ Gefährdet ist die Unternehmensreputation ${ }^{2}$ vor allem auf dem Kapitalmarkt, denn die Ad-hocPublizitätspflicht börsennotierter Unternehmen erfasst gerade auch potenziell rufschädigende Informationen. Denkbar ist sogar, dass eine Information kursrelevant und deshalb ad-hoc-pflichtig ist, weil sie das Reputationskapital des Unternehmens reduziert.

1 Zur Bedeutung und zu rechtlichen Aspekten des Reputationsmanagements SEIBT, DB 2015, 171; zur ökonomischen Reputationstheorie und rechtlichen Anwendungsfeldern KlÖHN/Schmolke, NZG 2015, 689.

2 Im Einklang mit der ökonomischen Reputationsforschung wird unter Unternehmensreputation im Folgenden verstanden die (1) auf dem bisherigen Verhalten der Gesellschaft beruhende (2) Einschätzung (3) der Stakeholder einer Gesellschaft, (4) inwieweit sich die Gesellschaft in Zukunft im Interesse der Stakeholder verhalten wird; vgl. Fombrun, Reputation: Realizing Value from the Corporate Image, 1996, S. 165: „a perceptual representation of a company's past actions and future prospects that describes the firm's appeal to all of its key constituents “; Fombrun/van Riel, 1 Corporate Reputation Review 5, 10 (1997): ,a collective representation of a firm's past actions and results that describes the firm's ability to deliver valued outcomes to multiple stakeholders"; RINDOva/WILLiamson/Petkova/Sever, 48 Academy of Management Journal 1033 (2005): „stakeholders' perception of an organization's ability to create value relative to competitors". 


\section{Beispielsfälle}

Dies zeigt Beispiel 1: Emittent $E$ produziert elektronische Haushaltsgeräte. Seine Erzeugnisse gelten als besonders hochwertig und lassen sich daher zu einem Premium-Preis verkaufen, der deutlich über dem Durchschnittspreis der Konkurrenzprodukte liegt. ${ }^{3}$ Besonders bekannt ist $E$ für seine Kühlschränke. Interne Tests ergeben, dass die zuletzt hergestellten Kühlschränke bei weitem nicht so langlebig sind wie vom Markt erwartet. Selbst wenn dadurch weder Gesetze verletzt wurden noch massenhafte Garantiefälle drohen, kann diese Information den Ruf des $E$ als Hersteller zuverlässiger Elektronikgeräte erheblich beschädigen und dazu führen, dass sich die Kunden von $E$ abwenden - und zwar nicht nur die Kühlschrankkäufer, sondern die Käufer aller von $E$ hergestellten Produkte. Schon die Erwartung solcher Absatzeinbrüche kann eine niedrigere Bewertung des $E$ rechtfertigen und die Kursrelevanz der Information über die internen Testergebnisse begründen.

Auch in Beispiel 2 spielt der Unternehmensruf eine wichtige Rolle: Die Chemiker von Emittent $E$, einem Pharmaunternehmen, haben Testergebnisse gefälscht, um die Zulassung für ein Medikament zu erhalten. Interne Untersuchungen der Compliance-Abteilung ergeben, dass es sich dabei um keinen Einzelfall handelt, sondern dass systematisch manipuliert und gefälscht wurde. Im Vorstand fragt man sich, ob man sofort eine Ad-hoc-Meldung veröffentlichen muss, da die bisherigen Erkenntnisse jedenfalls kursrelevant i.S.v. Art. 7 Abs. 1 lit. a) $\mathrm{MAR}^{4}$ sind.

\section{Bedeutung der Unternehmensreputation in der Praxis}

Ökonomische Studien haben ergeben, dass Aktionäre in Szenarien wie den Beispielsfällen den Reputationsverlust um ein Vielfaches stärker fürchten müssen als die rechtlichen Sanktionen - falls überhaupt solche Sanktionen drohen (vgl. Beispiel 1). So untersuchten die Ökonomen Jonathan Karpoff, Scott Lee und Gerald Martin 585 Emittenten, gegen die die Securities Exchange Commission (SEC) ein Verfahren wegen der Verletzung verschiedener Finanzberichtsvorschriften einleitete. Bereinigt um allgemeine Markteinflüsse gaben die

3 Zu den ökonomischen Grundlagen Klein/Leffler, 89 J. Pol. Econ. 615 (1981); Shapiro, 98 Q. J. Econ. 659 (1983); einführend KLÖHN/Schmolke, NZG 2015, 689, 690.

4 Verordnung (EU) Nr. 596/2014 des Europäischen Parlaments und des Rates vom 16. April 2014 über Marktmissbrauch (Marktmissbrauchsverordnung) und zur Aufhebung der Richtlinie 2003/6/EG des Europäischen Parlaments und des Rates und der Richtlinien 2003/124/EG, 2003/125/EG und 2004/72/EG der Kommission, ABl. EU Nr. L 173 v. 12.6.2014, S. 1. 
Aktienkurse der Emittenten im Zuge dieser Verfahren um durchschnittlich 38 \% nach. Diese Kursverluste bestanden aus drei Komponenten: (1) der Anpassung des Börsenkurses aufgrund der neuen Information (= der „Unwert der Fehlinformation"), (2) den erwarteten rechtlichen Sanktionen wie Schadensersatzklagen und Geldbußen sowie (3) den erwarteten Marktsanktionen, also dem Reputationsschaden 5 . Letzterer machte $2 / 3$ des gesamten Kursrückgangs aus. ${ }^{6}$ Ähnliche Ergebnisse erzielten andere Studien für das Vereinigte Königreich $^{7}$ und Japan ${ }^{8}$. Die Quintessenz lautet: Es sind meist nicht die rechtlichen Sanktionen, die der Verwaltung die Schweißperlen auf die Stirn treiben, wenn sie feststellt, dass Gesetze verletzt wurden, sondern der drohende Rufverlust.

\section{Kernfrage}

Damit gewinnt eine Frage an Bedeutung: Darf der Emittent die Ad-hocPublizität gem. Art. 17 Abs. 4 MAR aufschieben, um seinen Ruf zu schützen? Schon ein oberflächlicher Blick auf das Gesetz und seine Wertungen zeigt, wie schwierig die Antwort auf diese Frage ist.

Einerseits gehört der Ruf zu den wichtigsten Vermögensgegenständen des Emittenten. Deren Schutz ist legitimes Anliegen der Aktionäre und Zweck von Art. 17 Abs. 4 MAR. Andererseits haben die Aktionäre ein Interesse daran, von reputationsschädigenden Ereignissen und deren Hintergründen zu erfahren, damit sie ihre Kontrollrechte effektiver ausüben können. Könnte die Verwaltung die Ad-hoc-Publizitätspflicht aufschieben, um den Ruf des Emittenten zu schützen, würden die Aktionäre möglicherweise niemals erfahren, wie es tatsächlich um die Qualität und Integrität des Managements bestellt ist. Der Aufschub würde also nur vordergründig im Emittenteninteresse, tatsächlich aber im Interesse des Managements erfolgen. Auf lange Sicht könnten die Aktionäre deutlich mehr Geld verlieren als bei sofortiger Kapitalmarkttransparenz.

Bereits diese Überlegungen zeigen, dass in der Frage nach dem Aufschub der Ad-hoc-Publizität zwei Dimensionen des Unternehmensrufs wiederkehren,

5 Der Reputations(vermögens)schaden lässt sich daher definieren als der abgezinste Wert aller künftigen Mehrkosten und Mindereinnahmen der Gesellschaft, die darauf beruhen, dass die Stakeholder der Gesellschaft an der Fähigkeit oder Bereitschaft der Gesellschaft zur Einhaltung ihrer expliziten und impliziten Versprechen zweifeln; vertiefend KLÖHN/ Schmolke, NZG 2015, 689, 692f. Zur Abgrenzung von Reputationsvermögens- und bloßem Reputationsschaden noch u. II.4.

6 Karpoff/Lee/Martin, 43 J. Fin. \& Quant. Anal. 581, 582 (2008).

7 Armour/Mayer/Polo, Regulatory Sanctions and Reputational Damage in Financial Markets (March 9, 2015), Oxford Legal Studies Research Paper No. 62/2010, http://ssrn. com/abstract $=1678028$, sub. 5.2 und table 4.

8 Tanimura/Oкамото, 27 Asian Economic Journal 39, 52 (2013). 
die schon früh im ökonomischen Schrifttum herausgearbeitet wurden und seitdem im Zentrum der wirtschaftswissenschaftlichen Reputationsforschung stehen. ${ }^{9}$ Erstens ist er Disziplinierungsmittel, zweitens Vermögensgegenstand. ${ }^{10}$ Die Eigenschaft als Vermögensgegenstand spricht - jedenfalls auf kurze Sicht für den Aufschub der Ad-hoc-Publizität, die Eigenschaft als Disziplinierungsmittel spricht dagegen. Wie zu zeigen sein wird, kann man Art. 17 Abs. 4 MAR nur dann sachgerecht auslegen, wenn man diesen Widerspruch auflöst. ${ }^{11}$

\section{Ontologie und Abgrenzung}

Die rechtliche Analyse wird leichter, wenn man zuvor eine kleine Ontologie der typischen Sachverhalte skizziert, in denen sich die Frage nach dem Aufschub der Ad-hoc-Publizität zum Schutz des Unternehmensrufs stellt (s.u. 14). Sodann sind die hier behandelten Informationen von ähnlichen Informationen abzugrenzen, die ebenfalls ad-hoc-pflichtig sein können, häufig mangels Kursrelevanz aber nicht veröffentlichungspflichtig sind (5).

\section{Handelnder}

Grundsätzlich dürften die meisten Fälle wie folgt liegen: (1) Der Emittent oder die für ihn handelnden Personen (2) haben eine Handlung begangen, (3) die wenn sie öffentlich bekannt wird - (4) eine erhebliche Rufschädigung und daher einen Vermögensverlust des Emittenten zur Folge haben wird. Handelnde Personen können nicht nur die Organträger oder Arbeitnehmer des Emittenten sein, sondern prinzipiell alle Personen, deren Verhalten dem Emittenten unter Reputationsgesichtspunkten (!) zugerechnet wird, also alle Personen, für deren Verhalten der Markt zumindest auch den Emittenten abstraft. Möglich erscheinen solche Marktsanktionen etwa auch beim Verhalten von Tochtergesellschaften, Sub-Unternehmern oder Joint-Venture-Partnern des Emittenten. Die Frage der Ad-hoc-Publizität stellt sich freilich nur dann, wenn die Information den Emittenten unmittelbar i.S.v. Art. 17 Abs. 1 Satz 1 MAR betrifft, was selbst beim Handeln von Tochtergesellschaften nicht ohne Weiteres bejaht werden kann. ${ }^{12}$

9 Dazu m.w. N. Klöhn/Schmolke, NZG 2015, 689, $691 \mathrm{f}$.

10 Karpoff, in: Barnett/Pollock, The Oxford Handbook of Corporate Reputation, 2012, S. 361, 363. Vgl. auch Rindova/Martins, in: Barnett/Pollock, The Oxford Handbook of Corporate Reputation, 2012, S. 16, 24 f: „strategic intangible asset“.

11 S.u. III.

12 Allg. dazu KLÖHN, Kölner Komm. z. WpHG, 2. Aufl., 2014, $\ 15$ Rdn. 57 ff; speziell zur konzernrechtlichen Problematik KLÖHN, aaO, Rdn. $88 \mathrm{ff}$; monographisch demnächst Bartmann, Ad-hoc-Publizität im Konzern, Jur. Diss., München, 2016. 


\section{Rufschädigende Handlungen}

Als Handlung kommt grundsätzlich jedes Tun oder Unterlassen in Betracht, das den Ruf des Emittenten gefährdet. Klassischer Fall ist die Gesetzesverletzung. Wie Beispiel 1 zeigt, können Reputationsschäden aber auch abseits davon drohen.

\section{Feblende öffentliche Bekanntheit}

Die Frage nach dem Aufschub der Ad-hoc-Publizität stellt sich nur, solange die rufschädigende Information nicht öffentlich bekannt ist. Freilich sind hier Abstufungen denkbar und zwar gewissermaßen in zwei „Richtungen“. Auf „horizontaler" Ebene können von einer Gesamtheit rufschädigender Informationen sämtliche oder nur einzelne Informationen bekannt sein. In Beispiel 2 etwa mögen Manipulationen bei der Zulassung des Medikaments $A$ öffentlich bekannt sein, weitere Manipulationsfälle bei den Medikamenten $B, C$ und $D$ jedoch nicht. Sind sie kursrelevant, kann der Emittent zu weiteren Mitteilungen gem. Art. 17 Abs. 1 Satz 1 MAR verpflichtet sein. Auf „vertikaler“ Ebene kann man Informationen unterschiedlicher Verlässlichkeit über denselben Informationsgegenstand unterscheiden. ${ }^{13}$ So ist in Beispiel 2 denkbar, dass am Markt ein Gerücht über die Manipulationen bei Medikament $A$ kursiert. ${ }^{14}$ Weiß der Emittent, dass tatsächlich manipuliert wurde, oder muss er dies wissen, kann er zur Klarstellung im Wege der Ad-hoc-Meldung verpflichtet sein, wenn sein „Mehr“ an Gewissheit eine neue kursrelevante Information begründet. ${ }^{15}$

\section{Reputationsverlust}

Nicht jeder Reputationsschaden führt zu einem Vermögensschaden des Emittenten. Von den Reputationsvermögensschäden lassen sich die bloßen Reputationsschäden unterscheiden, die allein zu einem Ruf-, nicht aber zu einem Vermögensverlust führen. ${ }^{16}$ Letztere lösen für sich genommen keine Ad-hocPublizitätspflicht aus, weil es an der Kursrelevanz fehlt. Für die Abgrenzung kommt es darauf an, gegen wen sich das reputationsschädigende Verhalten des Emittenten richtet. Reputationsvermögensschäden entstehen nur dann, wenn

Zur Verlässlichkeit von Insiderinformationen konzeptionell KLÖHN, aaO (Fn. 12), $\ 13$ WpHG Rdn. $24 \mathrm{ff}$.

$14 \mathrm{Zu}$ Gerüchten als unsichere Informationen Fleischer/Schmolke, AG 2007, 841.

15 Dazu etwa KlöHn, aaO (Fn. 12), \$13 WpHG Rdn. 237.

16 Diese Terminologie vorschlagend KLÖHN/SChmolke, NZG 2015, 689, $692 \mathrm{f}$. 
die von dem Verhalten Betroffenen den Emittenten „abstrafen“ können, so dass er höhere Kosten oder geringere Einnahmen hat. In der ökonomischen Forschung werden diese Personen „related parties“ genannt, weil sie in einem besonderen Verhältnis zur Gesellschaft stehen (vor allem Aktionäre, Kreditgeber, Kunden, Lieferanten, Arbeitnehmer und Behörden). ${ }^{17}$

Betrifft das Verhalten keine konkreten Geschädigten oder können die Geschädigten nicht in der oben beschriebenen Weise reagieren (sog. „non-related parties“ oder "third parties“) $)^{18}$, bleibt der Vermögensschaden aus, weil die Gesellschaft allein aufgrund ihres Verhaltens weder höhere Kosten noch geringere Einnahmen befürchten muss. Unternehmen müssen z. B. mit erheblichen Vermögensverlusten rechnen, wenn sie ihre Kunden betrügen. Die Marktsanktion ist jedoch deutlich geringer oder entfällt sogar vollständig, wenn herauskommt, dass das Unternehmen gegen Exportbeschränkungen verstoßen hat. ${ }^{19}$

\section{Abgrenzung}

Von den hier interessierenden potenziell rufschädigenden Informationen sind verschiedene weitere Informationen abzugrenzen, die ebenfalls eine geringere Bewertung des Emittenten rechtfertigen und daher ad-hoc-pflichtig sein können.

- Erstens die nicht reputationsspezifischen Folgen der rufschädigenden Handlung. In Beispiel 2 etwa kann bereits die Information darüber, dass das Medikament nicht zulassungsfähig ist und daher vom Markt genommen werden muss, kursrelevant sein - unabhängig davon, auf welchen Gründen dies beruht.

- Zweitens die rechtlichen Sanktionen des rufschädigenden Verhaltens, also z.B. Geldstrafen ${ }^{20}$, Bußgelder, Schadensersatz- oder Rückrufpflicht.

17 Alexander, 42 J. L. \& Econ. 489, 491 (1999); Murphy/Shrieves/Tibbs, 44 J. Fin. \& Quant. An. 55, 56 (2009). In der eingangs zitierten Studie von Karpoff, LeE und MarTiN waren die Kapitalmarktteilnehmer unmittelbar von den Gesetzesverstößen betroffen, was auch das erhebliche Ausmaß der (Kapital-)Marktsanktion (Kursverlust) erklären mag.

18 Alexander, 42 J. L. \& Econ. 489, 491 (1999); Murphy/Shrieves/Tibbs, 44 J. Fin. \& Quant. An. 55, 56 (2009).

19 S. Alexander, 42 J. L. \& Econ. 489, 507-508 (1999); vgl. dazu allgemein Karpoff/LotT, 36 J. L. \& Econ. 757, 797 (1993); auf derselben Linie Karpoff/LotT/WehrLy, 48 J. L. \& Econ. 653, 668 (2005).

20 Freilich nur soweit der Emittent als juristische Person der Strafbarkeit unterliegt. 
- Drittens die Ursachen der rufschädigenden Handlung. So kann die rufschädigende Handlung auf höhere Agenturkosten im Emittenten schließen lassen, z. B. auf ein fehlerhaftes Compliance-System. ${ }^{21}$

Häufig werden diese Informationen, einzeln und kumulativ betrachtet, ${ }^{22}$ unterhalb der Kursrelevanzschwelle bleiben, so dass sich die Frage nach ihrer Ad-hoc-Publizitätspflicht gar nicht stellt. Selbst wenn sie kursrelevant und daher ad-hoc-pflichtig sind, kann der Emittent dennoch ein hohes Interesse an dem Aufschub der - hinzutretenden - rufschädigenden Information haben. Es kann einen erheblichen Unterschied machen, ob der Emittent dem Markt lediglich mitteilt, dass ein Produkt zurückgerufen wird, oder ob er bekannt gibt, der Rückruf des Produkts geschehe, weil Testergebnisse gefälscht wurden. Ebenso erscheint es nicht ausgeschlossen, dass der Emittent den Markt über ein Bußgeld oder andere verwaltungsrechtliche Sanktionen informiert, die Details des Sachverhalts aber in Absprache mit der Behörde geheim hält. Dies gelingt freilich nur, wenn im Hinblick auf die rufschädigende Information Art. 17 Abs. 4 MAR eingreift.

\section{Emittenteninteresse}

Die Befugnis zum Aufschub nach Art. 17 Abs. 4 MAR knüpft wie bisher ${ }^{23}$ an drei Voraussetzungen an: Der Aufschub muss erstens geeignet sein, das berechtigte Interesse des Emittenten ${ }^{24}$ zu beeinträchtigen (lit. a). Er darf zweitens nicht geeignet sein, die Öffentlichkeit irrezuführen (lit. b). Schließlich muss der Emittent die Geheimhaltung der Information sicherstellen können (lit. c). Die MAR übernimmt insoweit der Sache nach die bisherige Regelung in Art. 6 Abs. 2 MAD. Weitere materielle Regelungen über den Aufschub der Ad-hocPublizität enthält das neue Marktmissbrauchsrecht nicht. Auf Level 2 des Lamfalussy-II-Verfahrens ${ }^{25}$ sind lediglich technische Durchführungsstandards über die Mitteilungen im Zusammenhang mit dem Aufschub vorgesehen. ${ }^{26}$ Auf Level 3 hat die ESMA Leitlinien zu den berechtigten Emittenteninteressen

21 KlÖhn, ZIP 2015, 1145, 1151 f; zust. Wilken/Hagemann, BB 2016, 67, 69.

22 Zur erforderlichen Gesamtschau und zum Saldierungsverbot bei der Subsumtion unter \13 WpHG (jetzt Art. 7 MAR) KlöHn, aaO (Fn. 12), \13 WpHG Rdn. 45-47.

23 Bislang galt Art. 6 Abs. 2 der Richtlinie 2003/6/EG (Marktmissbrauchsrichtlinie), umgesetzt in $₫ 15$ Abs. 3 WpHG. Dazu sogleich im Text.

24 Art. 17 Abs. 4 lit. a) MAR stellt alternativ auf das berechtigte Interesse des Teilnehmers am Markt für Emissionszertifikate ab.

25 Zur Terminologie LutTer/Bayer/Schmidt, Europäisches Unternehmens- und Kapitalmarktrecht, 5. Aufl., 2012, $\mathbb{1} 17$ Rdn. 47; Walla, BKR 2012, 265, 267; dem folgend KLÖHN, in: Langenbucher, Europäisches Privat- und Wirtschaftsrecht, 3. Aufl., 2013, $\$ 6$ Rdn. 20.

26 Vgl. DurchführungsVO (EU) 2016/1055 v. 29.6.2016, ABl. EU Nr. L 173/47. 
herausgegeben, die einen Aufschub der Ad-hoc-Publizität rechtfertigen können. ${ }^{27}$ Die Frage des Reputationsschutzes wird darin jedoch nicht angesprochen.

\section{Emittenteninteresse als Aktionärsinteresse}

Man ist geneigt, in unseren Fällen die erste Voraussetzung des Art. 17 Abs. 4 MAR ohne viel Federlesens zu bejahen. Denn es scheint auf den ersten Blick kaum zweifelhaft, dass der Emittent ein Interesse am Schutz seines guten Rufes hat. Eine solche Betrachtung wäre jedoch voreilig. Dies zeigt sich, wenn man den Begriff des Emittenteninteresses in Art. 17 Abs. 4 MAR einmal genauer in den Blick nimmt.

Sowohl die ganz herrschende Ansicht in der Kommentarliteratur ${ }^{28}$ als auch die $\mathrm{BaFin}^{29}$ sind bisher davon ausgegangen, dass der Emittent ein „eigenes“ Interesse habe, das von dem Interesse seiner Stakeholder, insbesondere seiner Aktionäre, ${ }^{30} \mathrm{zu}$ unterscheiden sei. Wie dieses „eigene“ Emittenteninteresse zu bestimmen ist, wird, soweit ersichtlich, von niemandem näher erläutert. ${ }^{31}$ Diese konzeptionelle Offenheit bietet die Möglichkeit, eine ganze Reihe von Interessen zu definieren, die den Aufschub der Ad-hoc-Publizität rechtfertigen können - insbesondere das Interesse am Schutz der eigenen Reputation.

27 ESMA, Final Report Guidelines on the Market Abuse Regulation - market soundings and delay of disclosure of inside information, 13 July 2016, ESMA/2016/1130, krit. hierzu Krämer/Kiefner, AG 2016, 621.

28 Assmann, in: Assmann/Schneider, WpHG, 6. Aufl., 2012, $\mathbb{1 5}$ Rdn. 157; Zimmer/KruSE, in: Schwark/Zimmer, KMRK, 4. Aufl., 2010, $\$ 15$ Rdn. 58; Pfüller, in: Fuchs, WpHG, 2. Aufl., 2016, $\$ 15$ Rdn. 352; Косн, in: Veil, Europäisches Kapitalmarktrecht, 2. Aufl., 2014, $\ 19$ Rdn. 82f; PETSCH, Kapitalmarktrechtliche Informationspflichten versus Geheimhaltungsinteressen des Emittenten, 2012, S. 127; BitTer, WM 2007, 1953, 1960; Brandi/Süßmann, AG 2004, 642, 649f; Kersting, ZBB 2012, 442, 446; Pattberg/ BREDOL, NZG 2013, 87, 90; VeITH, NZG 2005, 254, 257.

29 BaFin, Emittentenleitfaden, 2013, S. 60 f.

30 Ausdrücklich in diesem Sinne Assmann, aaO (Fn. 28), $\mathbb{1} 15$ WpHG Rdn. 150; Pfüller, $\$ 15$ Rdn. 352; aaO (Fn. 28) Kersting, ZBB 2012, 442, 446.

31 Assmann, aaO (Fn. 28), $\mathbb{1} 15 \mathrm{WpHG}$ Rdn. 150 bietet eine Formel zur Bestimmung des Emittenteninteresses an, die die entscheidende Frage, woran der Emittent ein Interesse hat, offen lässt: „Allgemein ist deshalb ein berechtigtes Interesse des Emittenten am Aufschub einer Ad-hoc-Veröffentlichung dann anzunehmen, wenn mit überwiegender Wahrscheinlichkeit (...) die Veröffentlichung einerseits den Erfolg, den Eintritt oder die Durchführbarkeit eines Ereignisses, auf das sich die Insiderinformation bezieht, gefährden oder andererseits den Eintritt eines für den Emittenten negativen, aber durch geeignete Maßnahmen abwendbaren Ereignisses herbeiführen würde.“ Trotzdem soll die Veröffentlichung nicht deshalb aufgeschoben werden dürfen, weil sie unternehmerische Ziele vereiteln oder erheblich beeinträchtigen würde (!) (aaO, Rdn. 152). 
Eine solche Argumentation führt jedoch in die Irre. Im Gesellschaftsrecht darf es als gesichert gelten, dass Begriffe wie „Gesellschafts-“, „Unternehmens-" und daher auch Emittenteninteresse nicht wörtlich zu verstehen sind, sondern im Sinne abbrevierter Funktionsbegriffe, ${ }^{32}$ also als metaphorische Umschreibungen eines bestimmten juristischen Konzepts. Juristische Personen sind rechtliche Fiktionen. Sie können keine „eigenen“ Interessen haben. Wenn wir von Gesellschafts- oder Unternehmensinteresse sprechen, meinen wir die Interessen der hinter der Gesellschaft stehenden natürlichen Personen (Stichworte: shareholder value und stakeholder value). Auf welche Personen es für das Emittenteninteresse ankommen soll, beantwortet die h.M. aber gerade nicht. Das „eigene“ Interesse des Emittenten bleibt eine reine Fiktion - man kann prinzipiell jedes Interesse in diesen Begriff ,hineinlegen", auch solche Interessen, die tatsächlich nicht die des Emittenten, sondern des Vorstands, Aufsichtsrats oder des Mehrheitsaktionärs sind. Wie bereits erwähnt ${ }^{33}$, ist diese Gefahr in den hier behandelten Fällen besonders hoch: Häufig liegt die Vermeidung von Kapitalmarktpublizität vor allem im Interesse der Verwaltung, die möglicherweise gesellschaftsrechtliche Verantwortung für das rufschädigende Ereignis trägt und - sofern vorhanden - des Mehrheitsaktionärs, der nicht auf die Ad-hoc-Publizität angewiesen ist, um von dem reputationsschädigenden Vorfall zu erfahren, und der aufgrund des Handelserfordernisses nicht gem. $\$ \mathbb{S} 37 \mathrm{~b}, 37 \mathrm{c} \mathrm{WpHG}$ schadensersatzberechtigt ist.

Nach vorzugswürdiger Ansicht ist mit „Emittenteninteresse“ in Art. 17 Abs. 4 MAR das gemeinsame Interesse aller Aktionäre gemeint. ${ }^{34}$ Dies folgt nicht nur aus Erwägungsgrund Nr. $50 \mathrm{MAR}$, in dem ausdrücklich auf die „aktuellen und potenziellen Aktionäre" Bezug genommen wird, ${ }^{35}$ sondern auch aus dem Zweck von Art. 17 Abs. 4 MAR, der darauf gerichtet ist, die Geschäftschancen des Emittenten zu schützen und seine Investitionsanreize zu erhalten. ${ }^{36}$ Diese

32 Goette, FS 50 Jahre BGH, 2000, S. 123, 127 (im Hinblick auf den Begriff des „Unternehmensinteresses").

33 S. o. unter I.3.

34 Zuerst KLÖHN, ZHR 178 (2014), 55, 73 ff; DERs., aaO (Fn. 12), \15 WpHG Rdn. $190 \mathrm{ff}$; danach Voß, in: Jost/Voß/Ritz/Becker, WpHG, 2015, $\$ 15$ Rdn. 181; KLöHN/SCHMOLKE, NZG 2015, 689, 694; tendenziell auch Wilken/HagemanN, BB 2016, 67, 69, 71.

35 Mit „potenziellen Aktionäre[n]“ sind nicht etwa alle sonstigen Marktteilnehmer gemeint, sondern die Aktionäre, die während der Aufschubphase Aktionäre werden; s. zum gleichlautenden Art. 3 Abs. 1 lit. a) RL 2003/124/EG KLöHN, ZHR 178 (2014), 55, 75; DERs., aaO (Fn. 12), \15 WpHG Rdn. 191; Voß, aaO (Fn. 34), $\$ 15$ WpHG Rdn. 182.

36 S. wiederum KlöHN, ZHR 178 (2014), 55, 75f; DERs., aaO (Fn. 12), \15 WpHG Rdn. $193 \mathrm{f}$. 
Erkenntnis hat sich in anderen Rechtsordnungen ${ }^{37}$ bereits seit längerem durchgesetzt. $^{38}$

Das danach maßgebliche gemeinsame Interesse der Aktionäre ist auf die Maximierung des Fundamentalwerts ihrer Gesellschaft, also des Emittenten, gerichtet. ${ }^{39}$ In einem ersten Schritt können wir also festhalten: Der Aufschub i.S.d. Art. 17 Abs. 4 MAR ist im Interesse des Emittenten, wenn der Erwartungsnutzen des Aufschubs für einen möglichst hohen Fundamentalwert des Emittenten höher ist als der Erwartungsnutzen der (unverzüglichen) Veröffentlichung der Insiderinformation.

\section{Das Nutzenkalkül der Aktionäre zur Offenlegung reputationsschädigender Information}

\section{a) Interessenambivalenz}

Da die Aktionäre an einem möglichst hohen Fundamentalwert des Emittenten interessiert sind, ${ }^{40}$ sind sie in der Frage der (zügigen) Offenlegung rufschädigender Information zwiegespalten: Zwar möchten sie, dass das Reputationskapital des Emittenten möglichst erhalten bleibt. Je großzügiger der Vorstand die Ad-hoc-Publizität jedoch aufschieben darf, desto größer ist die Gefahr, dass sorgfalts- und treuwidriges Verhalten der Verwaltung vertuscht wird. Dies wiederum führt zu einem Anstieg der Agenturkosten und folglich zu einer Senkung des Fundamentalwerts des Emittenten. Die Aktionäre haben also ein ambivalentes Verhältnis zur Ad-hoc-Publizität: Einerseits wollen sie, dass das Reputationskapital möglichst erhalten bleibt. Andererseits wollen sie nicht auf die Disziplinierungswirkung der Ad-hoc-Publizität für Vorstand und Aufsichtsrat verzichten. Beide Wirkungen stehen in einem Spannungsverhältnis: Je höher der Ex-post-Schutz des Reputationskapitals, desto niedriger die Ex-ante-Disziplinierungswirkung der Ad-hoc-Publizität.

37 S. für das US-Recht etwa Kronfeld v. Trans World Airlines, Inc., 832 F.2d 726, 234 (2d Cir. 1987); aus dem Schrifttum Oesterle, 20 Cardozo L. Rev. 135, 204 (1998); LANGEvoORT, 98 Harv. L. Rev. 747, 791 (1985).

38 Ausführlich zum Ganzen KLÖHN, ZHR 178 (2014), 55, 73 ff; DERs., aaO (Fn. 12), \} 1 5 WpHG Rdn. $190 \mathrm{ff}$; s. ferner KLÖHN/Schmolke, NZG 2015, 689, 694.

39 Vgl. zum Shareholder-value-Ansatz nur Fleischer, in: Spindler/Stilz, AktG, 3. Aufl., 2015, $\$ 76$ Rdn. 19 m.w. N.

40 Zu den Einzelheiten KLÖHN, ZHR 178 (2014), 55, 73 ff. 


\section{b) Vergleichsmaßstab: Gebeimbaltung versus spätere Offenlegung}

Bei der Ermittlung des Emittenteninteresses ist zudem im Auge zu behalten, dass Art. 17 Abs. 4 MAR zumindest im Grundsatz nicht von der Veröffentlichung per se dispensiert, sondern lediglich einen (zeitweiligen) Aufschub der letztlich doch zu veröffentlichenden Information erlaubt. Anders verhält es sich nur, wenn sich die Information innerhalb der Aufschubperiode erledigt, etwa weil sie ihre Kursrelevanz verliert. ${ }^{41}$ Dann kann der Aufschub zur endgültigen, dauerhaften Nichtveröffentlichung der Information führen. Und nur wenn sich dies ex ante im Zeitpunkt der Aufschubentscheidung bereits abzeichnet $^{42}$, kann der Emittent für die Ermittlung seines Aufschubinteresses die zu erwartende Verringerung seines Fundamentalwerts durch die Veröffentlichung der reputationsschädigenden Information als solcher in Ansatz bringen. In allen anderen Fällen bestimmt sich das Interesse der Aktionäre an einem Aufschub der Veröffentlichung anhand eines Vergleichs des Erwartungsnutzens bzw. der erwartbaren Kosten der unverzüglichen Veröffentlichung mit den entsprechenden Auswirkungen der späteren Veröffentlichung. ${ }^{43}$

\section{c) Kosten der unverzüglichen Ad-boc-Publizität}

Für unseren Fall lässt sich zu den Kosten der unverzüglichen (und nicht erst späteren) Ad-hoc-Publizität Folgendes sagen.

\section{aa) Eindämmung des erwarteten Reputationsschadens durch Aufschub der Publizität}

Es kann im Interesse der Aktionäre liegen, die Ad-hoc-Publizität aufzuschieben, weil dadurch der erwartete Reputationsschaden eingedämmt wird. Schiebt der Emittent nicht auf, nimmt er diese Möglichkeit der Schadensreduktion nicht wahr. Die Kosten der unverzüglichen Ad-hoc-Publizität bestehen in dem nicht vermiedenen Verlust an Reputationskapital. Ob die Dinge so liegen, hängt von den genauen Umständen des Einzelfalls ab; pauschale Aussagen verbieten sich.

41 S. hierzu nur KLÖHN, aaO (Fn. 12), $\ 15$ WpHG Rdn. 325 zum noch geltenden $\ 15$ Abs. 3 Satz 3 und 4 WpHG.

42 Zur Maßgeblichkeit dieses Zeitpunkts s. nur Zimmer/Kruse, aaO (Fn. 28), \$15 WpHG Rdn. 56.

43 Hierauf unter dem Gesichtspunkt des Reputationsschadens hinweisend auch WiLKEN/ Hagemann, BB 2016, 67, 71. 
In unserem Beispiel 1 könnte ein solches Szenario wie folgt aussehen: Angesichts der Testergebnisse entscheidet sich das Management des $E$ unter Preisgabe eines Teils der Gewinnmarge künftig höherwertige Teile in den Kühlschränken zu verbauen. Zwar muss das genaue Zusammenspiel der neuen Komponenten noch von den Ingenieuren getestet werden. Man ist jedoch zuversichtlich, dass eher früher als später eine Lösung gefunden wird, welche die Langlebigkeit der Geräte erheblich erhöht. Sollte diese Lösung tatsächlich gelingen, so bilden die Testergebnisse nur noch die Situation bei den Altgeräten ab. Es erscheint plausibel, dass dies die reputationsschädliche Bewertung der Testergebnisse durch den Markt erheblich abmildert. Insofern läge ein Aufschub also im Emittenteninteresse.

Ebenso könnten in Beispiel 2 die internen Ermittlungen, die Verfolgung der Verantwortlichen und nicht zuletzt die Nachjustierung des Compliance-Systems erschwert werden, wenn der Emittent sofort an die Öffentlichkeit ginge und die Presse beginnt, über die Vorfälle zu berichten. Unter diesen Annahmen wäre der Reputationsschaden niedriger, wenn der Emittent die Ad-hocPublizität bis zur Reform der Compliance-Strukturen aufschieben würde.

\section{bb) Gefabr der Überreaktion des Marktes}

Der Aufschub nach Art. 17 Abs. 4 MAR kann außerdem kostensparend sein, wenn eine Überreaktion des Marktes auf die unverzügliche Offenlegung der reputationsschädigenden Information zu befürchten steht. ${ }^{44}$

Für unser Beispiel 1 ließe sich eine solche Überreaktion wie folgt denken: Die schlechten Testergebnisse für die Kühlschränke von $E$ sind keineswegs verallgemeinerungsfähig. Vielmehr bestehen durch Fakten untermauerte Anzeichen, dass die ansonsten von $E$ hergestellten Elektronikgeräte einen Langlebigkeitstest bestehen würden. So fällt jedenfalls die Einschätzung der Ingenieure von $E$ aus. Gleichzeitig weist die Marketingabteilung darauf hin, nach den bisherigen Marktbeobachtungen sei davon auszugehen, dass die Endabnehmer der Geräte des $E$ nach Bekanntwerden der negativen Testergebnisse diese nicht nur auf die Kühlschränke beziehen, sondern auch auf die anderen Produkte des $E$ übertragen werden. Bei hinreichendem zeitlichen Vorlauf könne man

44 S. dazu bereits den knappen Hinweis bei KlÖHN/Schmolke, NZG 2015, 689, 695; vgl. ferner Oesterle, 20 Cardozo L. Rev. 135, 224 (1998): „There is no doubt that the disclosure of bad news affects a firm's business reputation and the embarrassment alone of senior firm officials should not excuse the firm's obligation to be candid to the trading markets. But the disclosure of bad news has inherent dangers that, on occasion, can dwarf the value of the news itself. A negative disclosure can create its own reality as customers, suppliers, and employees panic and snowball a firm's troubles from distress to disaster." 
diese Reaktion jedoch durch Marketingmaßnahmen und Informationskampagnen abmildern.

In einem solchen Fall besteht auch ein Gleichlauf zwischen dem Aufschubinteresse des Emittenten und dem Interesse des Marktes, nicht durch die Information irregeführt zu werden. ${ }^{45}$ Das Aufschubinteresse wiegt folglich besonders schwer. Einen normativen Anker hat diese Überlegung in Erwägungsgrund 50 der MAR: In dem dort erwähnten zweiten Szenario wird für ein berechtigtes Aufschubinteresse darauf abgestellt, ob durch die unverzügliche Offenlegung der Information „die korrekte Bewertung der Information durch das Publikum gefährdet würde.“

Die Gefahr der Überreaktion spielt vor allem bei „unfertigen“, noch „weichen "Informationen eine Rolle, die sich durch weitere Sachverhaltsaufklärung weiter erhärten lassen. Sofern diese Informationen trotz aller Unsicherheiten bereits kursrelevant sind und unter Berücksichtigung des dem Emittenten zustehenden Prüfungsspielraums („sobald wie möglich“) bereits eine Veröffentlichungspflicht nach Art. 17 Abs. 1 UAbs. 1 MAR besteht ${ }^{46}$, stellt sich die Frage des Aufschubs nach Art. 17 Abs. 4 MAR. Zur Veranschaulichung diene erneut Beispiel 1: Die Testergebnisse für die Kühlschränke betrafen ein bestimmtes Modell. Einstweilen ist unklar, ob sie sich für die gesamte Modellpalette verallgemeinern lassen, oder ob die relative „Kurzlebigkeit“ nur die Kühlschränke des getesteten Modells betreffen. Der Vorstand von $E$ möchte dies zunächst klären, bevor er ,ad hoc geht“.

\section{cc) Reputationsbedingte Zusatzrendite wäbrend der Aufschubperiode}

Die sofortige Ad-hoc-Publizität führt noch auf andere Weise zu Kosten für die Aktionäre: Würde der Emittent die Offenlegung der Information aufschieben, könnte er während der Aufschubperiode weiterhin von den cash flows profitieren, die auf seiner (zu Unrecht) hohen Reputation beruhen. Bei unverzüglicher Offenlegung gingen diese cash flows verloren. Dieser Verlust führte zu einem vergleichsweise niedrigen Fundamentalwert.

In unserem Beispiel 1 könnte $E$ weiterhin einen Preisaufschlag auf seine Kühlschränke und sonstigen Elektronikgeräte verlangen, und zwar ohne etwas an den verwendeten Komponenten zu ändern, solange nur deren Ruf als qualita-

S. zum Marktinteresse noch unten unter IV.

46 Zur Unterscheidung zwischen den „Aufschubkonstellationen“ gem. Art. 17 Abs. 4 MAR und gem. Art. 17 Abs. 1 MAR s. ESMA, Consultation Paper Draft guidelines on the Market Abuse Regulation, 28 January 2016, ESMA/2016/162, Rdn. 67. Zur Frage der Sachaufklärungspflicht des Emittenten für die Bestimmung der Unverzüglichkeit i.S.d. $\ 15$ Abs. 1 Satz 1 WpHG a.F. s. ausführlich KlöHN, aaO (Fn. 12), 15 WpHG Rdn. $110 \mathrm{ff}$. 
tiv besonders hochwertig nicht durch die Bekanntmachung der Testergebnisse erschüttert wird. In Beispiel 2 verhält es sich ganz ähnlich: Solange die Fälschung der Testergebnisse nicht publik wird, kann das Pharmaunternehmen $E$ weiterhin (Zusatz-)Einkünfte generieren, die darauf beruhen, dass es weiterhin als zuverlässiger Entwickler wirksamer und zugleich unbedenklicher Medikamente angesehen wird.

Gegen die Berücksichtigung dieses „Aufschubnutzens“ bei der Ermittlung des Emittenteninteresses i.S.d. Art. 17 Abs. 4 MAR lassen sich jedoch zwei Einwände formulieren. Der erste Einwand ist ein tatsächlicher: Muss die Information nach Beendigung der Aufschubperiode wegen fortbestehender Kursrelevanz doch noch offengelegt werden oder gilt dies für eine damit zusammenhängende Information (Beispiel: Einbußen bei der Gewinnmarge wegen Einbau teurer Komponenten), so spricht viel dafür, dass der Emittent für sein „unehrliches“ Verhalten vom Markt noch einmal extra abgestraft wird." Die zeitweilige Extrarendite würde dann um den Preis eines sich verstärkenden Reputationsschadens erkauft. Der zweite - schlagende - Einwand ist ein normativer: Mit Blick auf das Publizitätsziel des Art. 17 MAR dürfte sich von selbst verstehen, dass man die Kosten der ausbleibenden Überrendite während der Aufschubperiode nicht in die Abwägungsentscheidung des Art. 17 Abs. 4 MAR einstellen darf. Denn insofern ist die Aufrechterhaltung einer fehlerhaften Markteinschätzung nicht bloße (zeitweilige) Nebenfolge des Aufschubs. Vielmehr zielt hier das Interesse der Aktionäre gerade auf die Aufrechterhaltung einer fehlerhaften Markteinschätzung zwecks Erwirtschaftung hierauf beruhender (!) (Zusatz-)Renditen. Dies kann nach der Teleologie des Art. 17 MAR kein anerkennenswertes („,berechtigtes“) Emittenteninteresse sein. ${ }^{48}$

\section{d) Nutzen der unverzüglichen Ad-boc-Publizität}

Das Nutzenkalkül der Aktionäre des von der reputationsschädigenden Information betroffenen Emittenten kann jedoch auch in Richtung einer unverzüglichen Offenlegung weisen. Denn diese ist nicht ausschließlich mit (zusätzlichen) Kosten verbunden, sondern kann auch Vorteile mit sich bringen. So kann die unverzügliche Offenlegung der Information im konkreten Fall helfen, das Ausmaß des Reputationsschadens zu begrenzen (aa.). Darüber hinaus kann die unverzügliche Ad-hoc-Publizität dazu beitragen, die Agenturkosten für die Aktionäre des Emittenten zu senken, und zwar unter zweierlei Gesichtspunkten (bb. und cc.). Überwiegen die Vorteile der sofortigen Ad-hocPublizität deren Nachteile, darf die Ad-hoc-Meldung schon mangels eines Emittenteninteresses an dem Aufschub, d.h. aus Gründen des Aktionärsschut-

S. bereits KlÖHN/SCHMOLKe, NZG 2015, 689, 695. 
zes, nicht aufgeschoben werden (Corporate Governance-Schranke ${ }^{49}$ des Art. 17 Abs. 4 MAR)..$^{50}$ Auf die weiteren Voraussetzungen von Art. 17 Abs. 4 MAR kommt es dann gar nicht an.

\section{aa) Vertiefung des Reputationsschadens durch verzögerte Offenlegung}

Die verzögerte Offenlegung hat nicht nur das Potenzial zur Senkung des Reputationsschadens. ${ }^{51}$ Vielmehr kann das Reputationsschadensrisiko auch steigen, weil die zeitweilige Geheimhaltung vom Markt als „Vertuschung“ wahrgenommen wird. Diese wird dann in mangelnde Vertrauenswürdigkeit übersetzt und bei der Neubewertung der über die Reputation ermittelten Eigenschaften des Emittenten „eingepreist“. ${ }^{52}$ Die zügige Information der Öffentlichkeit kann demgegenüber auch reputationsschadensmildernd wahrgenommen werden, nämlich als der erste Schritt zur Bekämpfung der Ursachen des Reputationsverlusts und damit als der erste Schritt zur Wiederherstellung der Reputation.

\section{bb) Agenturkosten überbewerteten Eigenkapitals}

Die unverzügliche Ad-hoc-Publizität reputationsschädigender Information kann im Interesse der Aktionäre sein, wenn und weil sich hierdurch die Agenturkosten senken lassen. Dies erklärt sich aus der folgenden allgemeinen Überlegung: Die Überbewertung des Eigenkapitals, also ein künstlich hohes Kursniveau, läuft dem Interesse der Aktionäre als Gruppe und damit dem Emittenteninteresse i.S.d. Art. 17 Abs. 4 MAR ganz allgemein zuwider, weil es deren Agenturkosten erhöht. ${ }^{53}$ Dies gilt jedenfalls dann, wenn man mit

49 Zur Terminologie KLÖHN, ZHR 178 (2014), 56, 85.

50 Zur aktionärsschützenden Funktion der Ad-hoc-Publizität SCHÖN, in: ders., Rechnungslegung und Wettbewerbsschutz im deutschen und europäischen Recht, 2009, S. 563, 579 f; Hellgardt, Kapitalmarktdeliktsrecht, 2008, S. 188f; Werner, Ein Publizitätskonzept, 2011, S. 150f; KLÖHN, ZHR 178 (2014), 55, 68 ff; DERs., ZIP 2015, 1145, 1149; SCHÖN, 6 J. Corp. L. Stud. 259, 273 (2006); zusf. KlöHN, aaO (Fn. 12), Vor $\ 15$ WpHG Rdn. $187 \mathrm{ff}$. In den USA Fleischer, 78 Harv. L. Rev. 1146, 1160-1168 (1965); danach etwa EasterBRook/Fischel, The Economic Structure of Corporate Law, 1991, S. 96 f; Seligman, 59 Brook. L. Rev. 1 (1993); Mahoney, 62 U. Chi. L. Rev. 1047 (1995); Langevoort, 79 Wash. U. L.Q. 449, 450 (2001); Thompson/Sale, 56 Vand. L. Rev. 859, 872 (2003); Goshen/ Parchomovsky, 55 Duke L.J. 711, 748 ff (2006). In Australien James Hardie Industries NV v. ASIC (2010) 81 ACSR 1, at [355]: „accountability of company management“.

51 S. dazu oben unter c) aa).

52 S. dazu bereits oben unter c) cc); dies nachdrücklich betonend auch WiLKEN/HagEMANN, BB 2016, 67, 71 .

53 Grundlegend Jensen, Fin. Mgmt. 34 (2005), 5 ff; s. ferner KlÖHN, ZHR 178 (2014), 55, $79 \mathrm{ff}, 81$; DERs., aaO (Fn. 12), $\$ 15$ WpHG Rdn. 202 m.w. N. Davon abgesehen wollen die Aktionäre die unverzügliche Offenlegung auch dann noch, wenn diese als Diszip- 
Michael Jensen eine solche Überbewertung danach bestimmt, ob es (nahezu) unmöglich ist, die fundamentale Leistungsfähigkeit des Unternehmens in einer Weise zu steigern, dass sie den gegenwärtigen Aktienkurs rechtfertigt. ${ }^{54}$ Denn das Management wird bestrebt sein, die unrealistisch hohen Erwartungen des Marktes zumindest kurz- bis mittelfristig nicht zu enttäuschen. Da die reale Leistungsfähigkeit des Unternehmens dies jedoch nicht hergibt, besteht die Gefahr, dass das Management nicht nachhaltige, sondern langfristig für das Unternehmen schädliche Strategien verfolgt. ${ }^{55}$ Spiegelt ein zu erwartender Reputationsschaden allein eine solche Überbewertung wider, dann stellt die mit der (unverzüglichen) Veröffentlichung einhergehende Kurskorrektur nur das ohnehin Unvermeidliche dar, zerstört also keine (echten) Werte. ${ }^{56}$

\section{cc) Agenturkosten bei Missmanagement und Compliance-Verstößen}

Betrifft die reputationsschädliche Information Missmanagement des amtierenden Vorstands oder Compliance-Probleme des Emittenten (wie in unserem Beispiel 2), so haben die Aktionäre regelmäßig kein Interesse an einer verzögerten Aufdeckung. Vielmehr wirkt die (prospektive) Marktsanktion nach (unverzüglicher) Veröffentlichung ex ante als Disziplinierungsmittel des Vorstands. Die Agenturkosten der Aktionäre werden auf diese Weise gesenkt. ${ }^{57}$ Es fehlt in diesen Fällen also grundsätzlich an einem Emittenteninteresse am Aufschub der Ad-hoc-Publizität.

Ausnahmsweise kann jedoch auch in derlei Fällen anderes gelten, also ein Aufschubinteresse des Emittenten bestehen. Dies ist dann der Fall, wenn die soeben beschriebene Senkung der Agenturkosten niedriger zu veranschlagen ist als die während der Aufschubperiode zu erwartende Senkung des Reputationsschadens. ${ }^{58}$ Eine solche Konstellation kann sich insbesondere dann er-

linierungsmittel so gut funktioniert, dass die Kosten der unverzüglichen Veröffentlichung niedriger ausfallen als die eingesparten Agenturkosten.

54 Jensen, Fin. Mgmt. 34 (2005), 5: „By definition, an overvalued equity means the company will not be able to deliver - except by pure luck - the performance to justify its value."

55 S. dazu wiederum Jensen, Fin. Mgmt. 34 (2005), 5. Weitere Agenturkosten einer Überbewertung können sich daraus ergeben, dass die Aktionäre bei einem höheren Börsenkurs weniger Anlass sehen, Kontrolle auszuüben, ferner aus einer höheren variablen Managervergütung.

56 In den Worten von Jensen, Agency Costs of Overvalued Equity, ECGI Working Paper $N^{\circ} .39 / 2004$, Abstract: „This value resetting (what I call the elimination of overvaluation) is not value destruction because the overvaluation would disappear anyway."

57 Einzelheiten dazu bei KLÖHN, ZHR 178 (2014), 55, 79ff, 81; DERs., aaO (Fn. 12), \} 1 5 WpHG Rdn. 202 m. w. N.; zust. Wilken/Hagemann, BB 2016, 67, 71.

58 S. in diesem Sinne bereits KlÖHN/SChMOlKe, NZG 2015, 689, 694; grds. zust. WilkeN/ Hagemann, BB 2016, 67, 71, die jedoch eine entsprechende Schadensvermeidung nur 
geben, wenn die konkrete Gefahr einer ganz erheblichen Marktüberreaktion bei unverzüglicher Offenlegung ${ }^{59}$ besteht.

Man stelle sich vor, dass die Missstände in unserem Beispiel 2 erst vom neuen Vorstand aufgedeckt werden. Dieser begibt sich auch postwendend daran, ein effektives Compliance-System zu installieren, um ähnliche Verhältnisse für die Zukunft möglichst auszuschließen. Müsste hier unverzüglich ad hoc gemeldet werden, bestünde die Gefahr, dass der Markt den Emittenten $E$ noch für Compliance-Verstöße über Reputationseinbußen abstraft, die mit dem Unternehmen in seiner aktuellen Verfassung nichts (mehr) zu tun haben (= Marktüberreaktion). Wenn der Vorstand in dieser Situation hinreichend plausibel darlegen kann ${ }^{60}$, dass der Agenturkostenvorteil der unverzüglichen Offenlegung auch in derlei (Compliance-)Fällen hinter dem mit einer übermäßigen Marktsanktion verbundenen Nachteil zurückbleibt, der sich durch geeignete Maßnahmen während der Aufschubperiode erwartungsgemäß abwenden lässt, würden auch die Aktionäre für einen Aufschub plädieren.

\section{e) Zwischenfazit}

Ob der (Erwartungs-)Nutzen für die Aktionäre als Gruppe höher ist als die Kosten, wenn der Emittent die Offenlegung einer reputationsschädigenden Information nach Art. 17 Abs. 4 MAR aufschiebt, ob ein solcher Aufschub also im Emittenteninteresse wäre, hängt damit maßgeblich von der Antwort auf folgende Fragen ab: (1) Besteht die konkrete Gefahr einer Überreaktion des Marktes, welcher durch Maßnahmen während der Aufschubperiode begegnet werden kann? (2) Lässt sich die Ursache des Reputationsschadens (überhaupt) beheben, so dass die Aufschubperiode genutzt werden kann, um den schließlich bei späterer Offenlegung auftretenden Reputationsschaden zumindest zu verringern? (3) Oder honoriert der Markt eine frühe Offenlegung der Information als Zeichen einer wirksamen und schnellen Ursachenbekämpfung, so dass geringere Reputationsverluste aufträten als bei einem Aufschub der Veröffentlichung?

dann annehmen, wenn „der Mantel des Schweigens [...] für alle Zeiten um den Compliance-Verstoß gehüllt werden“ kann. Die Marktsanktion qua Reputationsschaden nimmt die Aktionäre in ihrer Gesamtheit (!) hingegen nicht gegen eine unverzügliche Ad-hoc-Meldung ein, wenn diese Marktsanktion letztlich nur die „Überbewertung“ des Fundamentalwerts des Emittenten (wegen zu hoch eingeschätzter Managementqualität) aufzehrt. Dieser Verlust trifft die Aktionäre ohnehin, und dann lieber früher (einschließlich der damit verbundenen Senkung der Agenturkosten) als später. Dazu soeben unter aa).

59 Dazu soeben unter c) bb).

60 S. zur Plausibilisierungslast noch unten unter 3. 


\section{Aufschubentscheidung als Prognoseentscheidung des Vorstands}

Die Beantwortung dieser Fragen macht regelmäßig schwierige Prognoseeinschätzungen erforderlich. Für diese Aufgabe ist der Vorstand des Emittenten aufgrund seiner Funktion und Informationslage am besten geeignet. Es entspricht daher generell dem gemeinsamen Willen der Aktionäre, dass der Vorstand ex ante über das Vorliegen eines Aufschubinteresses des Emittenten entscheiden und die hierfür erforderlichen Prognoseeinschätzungen treffen sollte. ${ }^{61}$

Ebenso ist es jedenfalls grundsätzlich, d.h. vorbehaltlich entgegenstehender Interessenkonflikte in der Person des einzelnen Vorstandsmitglieds, ${ }^{62}$ im Interesse der Aktionäre, wenn der Vorstand diese Entscheidung „unbeschwert" treffen kann, d.h. ohne ein Haftungsrisiko aufgrund späterer Ex-post-Betrachtung durch den Richter fürchten zu müssen, der nunmehr unweigerlich das bekannte Ergebnis mitbedenkt (second-guessing in hindsight). Daher ist dem Vorstand - gerade auch im Interesse der Aktionäre in ihrer Gesamtheit ein gerichtlich nicht kontrollierbarer Beurteilungsspielraum zuzugestehen. ${ }^{63}$ Denn ebenso wie die durch $₫ 93$ Abs. 1 Satz 2 AktG geschützte unternehmerische Entscheidung im engeren Sinne ist die Entscheidung, ob ein Aufschub der Veröffentlichung nach Art. 17 Abs. 4 MAR im Emittenteninteresse (= Aktionärsinteresse) liegt, ,infolge ihrer Zukunftsbezogenheit durch Prognosen und nicht [oder jedenfalls nur eingeschränkt durch] justiziable Einschätzungen geprägt" ${ }^{64}$ Der Beurteilungsspielraum des Vorstands im Rahmen von Art. 17 Abs. 4 MAR ist dogmatisch freilich nicht mit einer analogen Anwendung des $\$ 93$ Abs. 1 Satz 2 AktG zu begründen. Vielmehr handelt es sich um eine daneben tretende Kategorie des Beurteilungsspielraums bei rechtlich gebundenen Vorstandsentscheidungen. ${ }^{65}$

Ungeachtet dieser dogmatischen Unterscheidung ergeben sich im Hinblick auf die Prüfkriterien „Überlappungen“ zwischen beiden Kategorien. ${ }^{66}$ Dies gilt namentlich für die angemessene Informationsgrundlage der Prognoseentscheidung. ${ }^{67}$ Freilich dürften die Pflichten zur Substantiierung der Tatsachenbasis,

61 KLÖHN, ZHR 178 (2014), 55, $85 \mathrm{ff}$.

62 S. dazu sogleich unter 4.a).

63 In allgemeinerem Zusammenhang bereits KLöHn, ZHR 178 (2014), 55, 85 ff.

64 S. Begr. RegE UMAG, BT-Drucks. 15/5092, S. 11 zur Regelung des $\ 93$ Abs. 1 Satz 2 AktG; dazu hier nur Fleischer, aaO (Fn. 39), \$93 AktG Rdn. 68.

65 S. zum Ganzen allgemein Fleischer, aaO (Fn. 39), $\$ 93$ AktG Rdn. 69 a m. w. N. zum Diskussionsstand; speziell zur Ermittlung des Emittenteninteresses i.S.d. $\$ 15$ Abs. 3 WpHG a.F. KLÖHN, ZHR 178 (2014), 55, 85 ff mit rechtsvergleichenden Hinweisen.

66 So wörtlich Fleischer, aaO (Fn. 39), \93 AktG Rdn. 69a.

67 S. dazu für Prognoseentscheidungen SpIndler, AG 2006, 677, 681; als Voraussetzung des $₫ 93$ Abs. 1 Satz 2 AktG Fleischer, aaO (Fn. 39), $₫ 93$ AktG Rdn. 70 ff. 
auf welcher die Einschätzungen des Vorstands über das Emittenteninteresse unter dem Gesichtspunkt des Reputationsschadens ruhen, ein ganzes Stück weiter reichen als im Anwendungsbereich des $\$ 93$ Abs. 1 Satz 2 AktG. ${ }^{68}$ Angesichts der Darlegungs- und Beweislast für das Emittenteninteresse in Art. 17 Abs. 4 MAR trifft den Vorstand eine flankierende Pflicht zur nachvollziehbaren und hinreichend ausführlichen Dokumentation dieser substantiierten Tatsachenbasis und der daran anknüpfenden Schlussfolgerungen. ${ }^{69}$ Dies betrifft namentlich den Hinweis auf konkrete Strategien zur Behebung von Reputationsschadensursachen während der Aufschubperiode und die Plausibilisierung ihrer Erfolgschancen ex ante.

In unserem Beispiel 1 müssten also nicht nur die Maßnahmen zur Ursachenbekämpfung (Ersatz minderwertiger Kühlschrankkomponenten durch hochwertige) oder zur Linderung einer erwarteten Überreaktion des Marktes (etwa durch Marketingmaßnahmen und Informationskampagnen) hinreichend dokumentiert, sondern auch ihre Erfolgsaussichten plausibilisiert werden. Für letzteres wären die erwähnten Einschätzungen von Entwicklungs- und Marketingabteilung einschließlich ihrer tatsächlichen Grundlagen zu erläutern. Auch wäre die Erwartung einer Überreaktion des Marktes näher zu begründen.

Diese Last zur Plausibilisierung der Erwartungen, sei es in Bezug auf die Senkung des Reputationsschadens(potenzials) während der Aufschubperiode, sei es in Bezug auf die Überreaktion des Marktes bei unverzüglicher Veröffentlichung, führt dazu, dass nicht jede noch so entfernte Möglichkeit ausreicht, um ein Aufschubinteresse zu begründen.

Allein aufgrund seiner aktienrechtlichen Pflichtenbindung muss der Vorstand bei seiner Prognoseentscheidung selbstverständlich auch „zum Wohle der Gesellschaft“ und „in gutem Glauben“ handeln. ${ }^{70}$ Aus diesen allgemeinen

68 S. für $₫ 93$ Abs. 1 Satz 2 AktG wiederum Fleischer, aaO (Fn. 39), $\ 93$ AktG Rdn. 70 ff mit Hinweis auf die „Relevanz von Bauchentscheidungen“, sowie Begr. RegE UMAG BT-Drucks. 15/5092, S. 11, wo für unternehmerische Entscheidungen auf die Bedeutung von „Instinkt, Erfahrung, Phantasie und Gespür für künftige Entwicklungen und ein[...] Gefühl für die Märkte“ verwiesen wird; vgl. demgegenüber zur Skepsis gegenüber einem pauschalen Hinweis des Aufsichtsrats auf mögliche Reputationsschäden zur Rechtfertigung der Nichtverfolgung von Haftungsansprüchen gegenüber dem Vorstand KLÖHN/Schmolke, NZG 2015, 689, 694 m. w. N.; vgl. ferner OLG Düsseldorf AG 2010, 126, 130 zur Darlegung negativer Auswirkungen einer Sonderprüfung.

69 Anders als im Rahmen des $₫ 93$ Abs. 1 Satz 2 AktG (s. dafür Fleischer, aaO (Fn. 39), \93 AktG Rdn. 77) handelt es sich vorliegend um eine Organpflicht, da den Nachteil der Nichterweislichkeit nicht (nur) der Vorstand (wie bei $\int 93 \mathrm{AktG}$ ), sondern zunächst und vor allem der Emittent, also die AG trägt.

70 S. zu diesen Vorgaben bei $\$ 93$ Abs. 1 Satz 2 AktG nur Fleischer, aaO (Fn. 39), $\$ 93$ AktG Rdn. $73 \mathrm{ff}$. 
äußeren Grenzen des Vorstandsermessens dürfte sich immerhin für Art. 17 Abs. 4 MAR ergeben, dass ein Aufschub reputationsschädlicher Information im Emittenteninteresse nur in Betracht kommt, wenn der Vorstand die Ursachen „bei der Wurzel zu packen“ gedenkt und nicht lediglich kosmetische Maßnahmen ins Auge fasst.

\section{Entscheidung durch einen befangenen Vorstand}

\section{a) Beurteilungsspielraum und Vorbehalt der Befangenheit}

Dass der Vorstand im Rahmen der Entscheidung über den Aufschub nach Art. 17 Abs. 4 MAR grundsätzlich einen Beurteilungsspielraum bei der Frage hat, ob der Aufschub im Emittenteninteresse liegt, folgt - wie gesehen - aus dem Zweck und Begriff des Emittenteninteresses. ${ }^{71}$ Da mit dem Emittenteninteresse das gemeinsame Aktionärsinteresse gemeint ist und da die Aktionäre ein Interesse daran haben, dass der Vorstand und nicht die Gerichte die schwierige Abwägung der Vor- und Nachteile der unverzüglichen Ad-hocPublizität vornehmen, unterliegt die Frage, ob der Aufschub im Emittenteninteresse war, nicht der vollen gerichtlichen Überprüfung.

Folgt man dieser Begründung, erkennt man schnell, dass der Beurteilungsspielraum unter einem Vorbehalt steht, der aus der Dogmatik zur Business Judgment Rule bekannt ist: Über den Aufschub muss ein desinteressierter, d. h. ein unbefangener Vorstand entscheiden. ${ }^{72}$ Entscheidet ein befangener Vorstand mit Sonderinteressen, besteht die konkrete Gefahr, dass er sich bei seiner Entscheidung nicht ausschließlich am Wohl der Aktionäre orientiert. In diesem Fall wollen die Aktionäre gerade keinen Beurteilungsspielraum des Vorstands. Vielmehr ist es in ihrem Sinne, dass die Gerichte die Frage, ob der Aufschub nach Art. 17 Abs. 4 MAR im Emittenteninteresse liegt, vollumfänglich überprüfen.

Dieses Szenario ist in den hier behandelten Fällen gut denkbar. Man stelle sich nur vor, sämtliche Vorstandsmitglieder, die in Beispiel 2 über den Aufschub der Ad-hoc-Publizität entscheiden, waren bereits im Amt und wussten von den Gesetzesverletzungen. Es bedarf kaum der Erklärung, dass sich diese Mitglieder bei der Entscheidung darüber, ob der Emittent möglichst viele Informationen geheimhalten oder die Publizität des Kapitalmarktes früh suchen sollte, nicht nur am Interesse der Aktionäre orientieren könnten, sondern auch

71 S. dazu ausführlich oben unter 1.

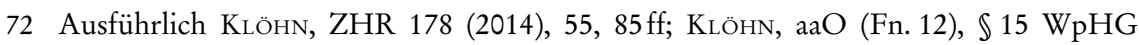
Rdn. 220 ff; vgl. zur Business Judgment Rule gem. \93 Abs. 1 Satz 2 AktG Begr. RegE UMAG, BT-Drucks. 15/5092, S. 11: Das Handeln des Vorstands muss „unbeeinflusst von Interessenkonflikten, Fremdeinflüssen und ohne unmittelbaren Eigennutz“ sein. 
an ihrem Eigeninteresse, die Haftung gegenüber der Gesellschaft und weitere gesellschaftsrechtliche Konsequenzen zu vermeiden. Aufgeworfen sind damit zwei Fragen: Wann besteht ein Sonderinteresse, das die volle gerichtliche Überprüfung der Aufschubentscheidung rechtfertigt (s.u. b)? Was sind die genauen Konsequenzen einer solchen Befangenheit (c)?

\section{b) Sonderinteresse des Vorstands}

a) Maßstab

Wann ein Sonderinteresse vorliegt, welches den Beurteilungsspielraum entfallen lässt, ergibt sich relativ zwanglos aus den bisherigen teleologischen Überlegungen. $\mathrm{Da}$ Grund des Beurteilungsspielraums das Aktionärsinteresse ist und auch die Grenzen des Beurteilungsspielraums aus dem Aktionärsinteresse abzuleiten sind, genießt der Vorstand keinen Beurteilungsspielraum, wenn konkrete Umstände vorliegen, die befürchten lassen, dass er die Entscheidung über den Aufschub der Ad-hoc-Publizität nicht allein im Interesse der Aktionäre treffen wird. Anhaltspunkte zur Konkretisierung dieses Maßstabs findet man in der Dogmatik zum „Sonderinteresse“ bei der Business Judgment Rule einerseits $^{73}$ und zum Stimmrechtsausschluss des GmbH-Gesellschafters wegen Befangenheit (vgl. $\$ 47$ Abs. 4 GmbHG) andererseits ${ }^{74}$.

\section{bb) Konkrete Tatsachen}

Erforderlich sind konkrete Verdachtsmomente für einen Interessenkonflikt. Allgemein-abstrakte Befürchtungen, der Vorstand sei befangen, genügen nicht. Wäre dies anders, würden Vorstände schon bei geringsten Zweifeln an ihrer Unbefangenheit zu einer möglichst frühzeitigen Veröffentlichung der ad-hocpflichtigen Information neigen, um ihre Rückgriffshaftung gem. $\mathbb{S} 93$ Abs. 2 Satz 1 AktG gegenüber dem Emittenten zu vermeiden. Solche abstrakten Zweifel ließen sich zudem fast immer konstruieren, wenn man die ökonomische Grundannahme eigennützig handelnder Akteure ernst nimmt. Das liegt aber nicht im Interesse der Aktionäre. Anders ist dies, wenn sich die Sorge um die Befangenheit auf konkrete Tatsachen stützt.

\section{cc) Insbesondere: Haftungsrisiken des Vorstands}

Die bloße Tatsache, dass der Vorstand, der über die Ad-hoc-Publizität (mit-) entscheidet, zum Zeitpunkt der reputationsschädigenden Handlung bereits im Amt war und daher grundsätzlich gesellschaftsrechtliche Mitverantwortung 
tragen könnte, genügt daher nicht, um den Beurteilungsspielraum zu verneinen. Anders ist dies, wenn konkrete Tatsachen nahelegen, dass der Vorstand gegenüber der Gesellschaft haften könnte. Je höher die potenzielle Haftung des Vorstands, desto berechtigter ist ceteris paribus die Sorge der Aktionäre, dass der Vorstand nicht unbefangen über den Aufschub der Ad-hoc-Publizität entscheiden kann. Bei höherem Haftungspotenzial können bereits niedrigere Anforderungen an den betreffenden Verdacht ausreichen, um eine Befangenheit des Vorstands zu bejahen.

\section{dd) Drittinteressen und Konzerninteresse}

Welche Interessen jenseits des angesprochenen Vorstandsinteresses an einer Haftungsvermeidung als „Befangenheitsgründe“ taugen, lässt sich kaum allgemein bestimmen. Sie müssen gemäß der eingangs vorgestellten Testfrage so beschaffen sein, dass sie die Befürchtung nahelegen, der Vorstand orientiere sich bei der Entscheidung über den Aufschub der Ad-hoc-Publizität nicht allein am Wohl der Aktionäre. Hierbei kommen nicht nur Eigen-, sondern auch Drittinteressen in Betracht, etwa die Interessen nahestehender Personen oder - in praxi wohl relevanter - die Interessen des Groß- oder Mehrheitsaktionärs, dessen Interessen wiederum von den Interessen aller Aktionäre an der Maximierung des Fundamentalwerts abweichen können.

Besonderheiten gelten in der faktischen Unternehmensverbindung, weil die $\mathbb{S} 311 \mathrm{ff}$ AktG bekanntlich nicht nur die abhängige Gesellschaft schützen, sondern auch eine Konzernermöglichungsfunktion haben. ${ }^{75}$ In unserem Kontext bedeutet dies, dass der Vorstand der abhängigen AG die Ad-hoc-Publizität auf Veranlassung des herrschenden Unternehmens aufschieben darf, wenn dies zwar für den Emittenten nachteilig, aber im Interesse des herrschenden Unternehmens ist und auch die weiteren Voraussetzungen des $\mathbb{3} 311 \mathrm{AktG}$ erfüllt sind. Es gilt insoweit nichts anderes als bei der Weitergabe von Insiderinformationen in der faktischen Unternehmensverbindung, denn auch sie kann im Konzerninteresse gerechtfertigt sein. ${ }^{76}$

\311 AktG setzt für eine konzernrechtliche Zulässigkeit neben dem bestehenden Konzerninteresse voraus, dass (1) der Nachteil ausgleichsfähig ist und (2) von einem späteren Nachteilsausgleich auch tatsächlich ausgegangen werden

75 Zur Konzernermöglichungsfunktion der $\int \mathbb{S} 311 \mathrm{ff}$ AktG Altmeppen, Münchner Komm.

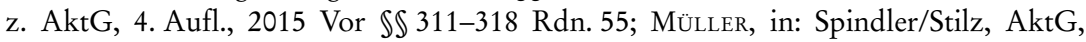
3. Aufl., 2015, Vor $\$ \mathbb{S} 311-318$ Rdn. 37; K. SснміDт, Gesellschaftsrecht, 4. Aufl., 2002, \} 1 7 \text { IV 1; MÜlberT, Aktiengesellschaft, Unternehmensgruppe und Kapitalmarkt, } 2. Aufl., 1996, S. 16, 452; Verse, Der Gleichbehandlungsgrundsatz im Recht der Kapitalgesellschaften, 2006, S. 340.

76 Dazu Klöhn, aaO (Fn. 12), \14 WpHG Rdn. 380 ff; Verse, aaO (Fn. 75), S. 528; Uwe H. SCHNeIDER, FS Wiedemann, 2002, S. 1255, 1267. 
kann..7 $\mathrm{Ob}$ erwartete Reputationsschäden des abhängigen Emittenten überhaupt in diesem Sinne ausgleichsfähig sein können, hängt entscheidend davon $a b$, welche Anforderungen man an die Quantifizierbarkeit des Nachteils, insbesondere an prognostische Bewertungen stellt. ${ }^{78} \mathrm{Können} \mathrm{diese} \mathrm{konzernrecht-}$ lichen Hürden genommen werden, darf die Ad-hoc-Publizität freilich nicht allein deshalb aufgeschoben werden, weil dies i.S.d. herrschenden Unternehmens ist. Vielmehr sind die weiteren Voraussetzungen des Art. 17 Abs. 4 MAR zu beachten.

Zur Veranschaulichung diene wiederum eine Abwandlung unseres Beispiels 1: Nehmen wir an, Emittent $E$ sei eine abhängige Gesellschaft des herrschenden Unternehmens $U$. Der Vorstand von $E$ kommt zu dem Ergebnis, dass eine unverzügliche Ad-hoc-Meldung im Interesse von $E$ sei, weil die zeitweilige Geheimhaltung vom Markt als „Vertuschung“ wahrgenommen und daher den entstehenden Reputationsschaden vertiefen würde. ${ }^{79}$ Der Vorstand von $U$ teilt $E$ jedoch mit, dass ein Aufschub der Offenlegung im Konzerninteresse wäre, weil eine andere Konzerntochter gerade ebenfalls Qualitätsprobleme habe und das zeitgleiche Bekanntwerden der Qualitätstests bei $E$ womöglich dazu führte, dass der Markt von einem übergreifenden „Konzernproblem“ mit entsprechenden Konsequenzen für andere Tochterunternehmen ausgehe. Er erwarte daher ein entsprechendes Verhalten seitens des Vorstands von $E$.

Begründet der Vorstand der abhängigen Gesellschaft den Aufschub in solchen Fällen mit dem Konzerninteresse (scil. dem Interesse von $U$ ), dann prüft das Gericht also unter dem Gesichtspunkt des Emittenteninteresses die Voraussetzungen des $\mathbb{3} 311$ AktG. Im Hinblick auf die gerichtliche Überprüfungsdichte der Entscheidung kommt es dann darauf an, inwieweit man dem Vorstand im Rahmen der Entscheidung nach $\$ 311$ AktG einen Beurteilungsspielraum zubilligt. ${ }^{80}$

Aus europäischer Perspektive ist nach dem Gesagten also von einem „konzern(rechts)offenen" Begriff des Emittenteninteresses in Art. 17 Abs. 4 MAR auszugehen. Dies liegt auch in der Fließrichtung der Rechtsentwicklung auf Unionsebene, wo sich die Kommission um eine EU-weite Anerkennung des Gruppeninteresses bemüht. ${ }^{81}$

77 Habersack, in: Emmerich/Habersack, Aktien- und GmbH-Konzernrecht, 8. Aufl., 2016, $\ 311$ Rdn. 60, 78; s. auch KocH, in: Hüffer, AktG, 12. Aufl., 2016, $\$ 311$ Rdn. 48 jew. m.w.N.

78 S. zur Diskussion nur Koch, aaO (Fn. 77), $\$ 311$ AktG Rdn. 39 m.w.N.

79 S. zu dieser Fallkonstellation allgemein oben unter 2. d) aa).

80 Dies ist streitig. S. zum Meinungsstand nur KocH, aaO (Fn. 77), $\$ 311$ AktG Rdn. 39 m.w.N.

81 Letzter Stand ist die Ankündigung der Kommission aus dem Jahre 2012 eine Initiative für eine bessere Anerkennung des Begriffs „Gruppeninteresse“ im Jahr 2014 vorzulegen (Dok. KOM (2012) 740, S. 17 Rdn. 4.6); der Europäische Wirtschafts- und Sozialaus- 


\section{ee) Beurteilungsperspektive}

$\mathrm{Ob}$ der Vorstand befangen ist, ist aus einer objektiven Ex-ante-Perspektive zum Zeitpunkt der Aufschubentscheidung zu beurteilen. Stellt sich später heraus, dass kein Sonderinteresse vorlag, ist dies für sich genommen irrelevant. Entscheidend ist, ob ex ante eine sachwidrige Entscheidung zu befürchten war. Ein Sonderinteresse kann also auch dann vorliegen, wenn der Vorstand sich dieses Interesses zum Zeitpunkt der Entscheidung nicht bewusst ist ${ }^{82}$ und sich der Interessenkonflikt damit letztlich auch nicht in der konkreten Entscheidung niederschlägt.

\section{c) Rechtsfolgen bei Befangenheit des Vorstands}

Ist der Vorstand nach den dargestellten Maßstäben befangen, hängen die Rechtsfolgen von seinem weiteren Verhalten ab: Soll der grundsätzlich bestehende Beurteilungsspielraum für die Ermittlung des Emittenteninteresses erhalten bleiben, dürfen die befangenen Organwalter bei der Aufschubentscheidung nicht mitwirken (s.u. aa). Tun sie es doch, entfällt der Beurteilungsspielraum bei der Frage, ob der Aufschub im Interesse des Emittenten liegt. Diese Frage unterliegt dann also der vollen gerichtlichen Überprüfung (s.u. $\mathrm{bb})$.

\section{aa) Mitwirkungsverbot}

Ist der Vorstand befangen, darf er nicht an der Entscheidung über den Aufschub der Ad-hoc-Publizität mitwirken. Dies bedeutet nicht nur, dass er von der eigentlichen Entscheidung ausgeschlossen ist, sondern auch, dass er in keiner anderen Weise an der Entscheidung über den Aufschub mitwirken darf, auch nicht durch seine Teilnahme an den Sitzungen und den Diskussionen, die der Aufschubentscheidung vorangehen.$^{83}$ Denn nach den oben herausgearbeiteten Grundsätzen entfällt der Beurteilungsspielraum, wenn und weil die Aktionäre befürchten müssen, dass die Entscheidung über den Aufschub der Ad-hoc-Publizität nicht in ihrem Interesse erfolgt. Diese Befürchtung ist nicht nur dann gerechtfertigt, wenn der Vorstand förmlich über den Aufschub ent-

schuss hat sich in einer Stellungnahme allerdings gegen die Anerkennung eines Gruppeninteresses ausgesprochen. S. ABl. EU v. 19.9.2013, Nr. C 271/70 auf S. 70, 74. Vgl. dazu auch Habersack, Münchener Komm. z. AktG, 4. Aufl., 2016, Einleitung Rdn. 109; Müller, aaO (Fn. 75), Vor $\iint 311-318$ AktG Rdn. 20.

82 Freilich kann der Rückgriff der Gesellschaft gem. $₫ 93$ Abs. 2 Satz 1 AktG in diesem Fall am fehlenden Verschulden des Vorstands scheitern.

83 Anders die hM zu $₫ 47$ Abs. 4 GmbHG, vgl. nur Casper, in: Bork/Schäfer, GmbHG, 3. Aufl., 2015, $\$ 47$ Rdn. 42; Drescher, aaO (Fn. 74), \47 GmbHG Rdn. 136. 
scheidet, sondern auch dann, wenn er sich auf andere Weise in die Entscheidungsfindung einbringt.

Auf dieser Grundlage lassen sich auch Fälle lösen, in denen das befangene Vorstandsmitglied gemeinsam mit anderen über den Aufschub abstimmt, seine Stimme für den Aufschub jedoch nicht kausal wurde, die anderen unbefangenen Vorstandsmitglieder die Publizität also auch ohne seine Stimme aufgeschoben hätten. Entscheidend ist nicht, dass die Stimme des Befangenen nicht kausal war. Entscheidend ist, ob zu befürchten ist, dass er die anderen Vorstände mitbeeinflusst hat. Nur wenn sich dies mit Sicherheit ausschließen lässt, wenn der Entscheidungsfindungsprozess also nachweislich „frei“ vom Einfluss des befangenen Mitglieds war, ist ein Beurteilungsspielraum gerechtfertigt.

Haben sämtliche Vorstandsmitglieder Sonderinteressen, so dass eine unbefangene Entscheidung über den Aufschub der Ad-hoc-Publizität im Vorstand nicht möglich ist, muss der Aufsichtsrat über den Aufschub entscheiden, da dies dem Interesse der Aktionäre am besten entspricht. ${ }^{84}$ Der Aufsichtsrat genießt bei seiner Entscheidung unter denselben Voraussetzungen einen Entscheidungsspielraum wie der Vorstand. Sind auch sämtliche Aufsichtsratsmitglieder befangen, ist eine Aufschubentscheidung mit Beurteilungsspielraum nur möglich, wenn zumindest ein unbefangener Entscheidungsträger (Vorstand oder Aufsichtsrat) bestellt bzw. ernannt wird respektive die durch zwingende Vorgaben der Beschlussfähigkeit erforderliche Anzahl an Entscheidungsträgern ${ }^{85}$.

\section{bb) Volle gerichtliche Überprüfung}

Wirkt ein befangener Vorstand an der Aufschubentscheidung mit, und ist daher zu befürchten, dass diese Entscheidung nicht allein im Interesse der Aktionäre erfolgte, so steht dies einem zulässigen Aufschub der Ad-hocPublizität nicht per se entgegen. Jedoch unterliegt die Frage, ob diese Entscheidung im Emittenten(= Aktionärs-)interesse lag, in diesem Fall der vollen gerichtlichen Überprüfung. ${ }^{86}$

Das Gericht prüft also, ob der Aufschub der Ad-hoc-Publizität einen höheren Nutzen für die Aktionäre versprach als die unverzügliche Offenlegung und beschränkt sich dabei nicht auf eine Plausibilitätsprüfung. Im Rahmen dieser Prüfung berücksichtigt das Gericht nicht nur die erwarteten Auswirkungen

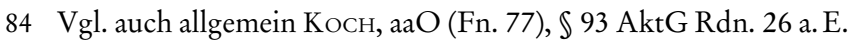

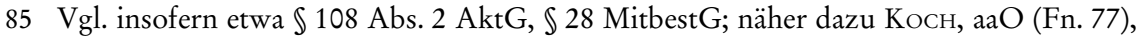
$§ 108$ AktG Rdn. $15 \mathrm{ff}$.

86 Vgl. auch allgemein und im Einzelnen abweichend Косн, aaO (Fn. 77), $\mathbb{9} 93$ AktG Rdn. 26 und für den Aufsichtsrat $\$ 108$ Rdn. 13 jew. m.w. N. 
der Geheimhaltung auf den drohenden Reputationsschaden (Eindämmung oder Vertiefung?), sondern auch, mit welchem Verlust an Disziplinierungswirkung der Ad-hoc-Pflicht es verbunden ist, wenn Vorstände sie in Situationen wie dem konkret vorliegenden Fall aufschieben dürfen (ökonomisch ausgedrückt: Inwieweit der Aufschub der Ad-hoc-Publizität die Agenturkosten für die Aktionäre des Emittenten erhöht). ${ }^{87}$

Dass diese Kosten-Nutzen-Rechnung nicht mit mathematischer Präzision durchgeführt werden kann, bedarf keiner näheren Ausführung. Selbstverständlich lassen sich die einzelnen Posten nur grob abschätzen. Die Besonderheit besteht darin, dass das Gericht diese Schätzung im vollen Umfang selbst vornimmt, während unbefangene Vorstände einen Beurteilungsspielraum genießen.

Wie gesehen, kann die Kosten-Nutzen-Rechnung auch dann zugunsten der Geheimhaltung ausschlagen, wenn ein konkretes Fehlverhalten des Vorstands im Raume steht, der über die Ad-hoc-Publizität mitentscheidet. Nur dürfte dies auf Ausnahmesituationen begrenzt sein. ${ }^{88}$

\section{Weitere Voraussetzungen von Art. 17 Abs. 4 MAR}

Ist die (zeitweilige) Geheimhaltung der reputationsschädigenden Information im Interesse des Emittenten, ist nur die erste Hürde des Art. 17 Abs. 4 MAR übersprungen. Selbstverständlich müssen die weiteren Voraussetzungen dieser Norm erfüllt sein, damit die Ad-hoc-Publizität aufgeschoben werden kann, der Emittent muss also die Geheimhaltung der Information gewährleisten können und es darf keine Irreführung des Marktes zu befürchten sein. ${ }^{89} \mathrm{Da}$ es bei diesen Voraussetzungen um das Marktinteresse geht, genießt der Vorstand bei der Prüfung dieser Voraussetzungen keinen Beurteilungsspielraum. ${ }^{90} \mathrm{Da}$ diese Voraussetzungen keine reputationsspezifischen Fragen aufwerfen, sollen sie hier ausgeblendet werden.

$87 \mathrm{Zu}$ allen Einzelheiten o. 2.

88 S.o. 2. d) cc)?

89 Die weitere Voraussetzung eines überwiegenden Emittenteninteresses (so wie es $\mathbb{6} 6$ WpAIV kurioserweise noch immer unter Verweis auf $\$ 15$ Abs. 3 WpHG verlangt), ist mit der MAR entfallen; vgl. dazu und zu dem fortbestehenden $\ 6$ WpAIV KLÖHN, AG 2016, 423, 430f. zust. Poelzig, NZG 2016, 761, 764; Kumpan, DB 2016, 2039, 2043. Dies dürfte auch der Ansicht der ESMA entsprechen, die in ihren Level-3-Leitlinien zu Art. 17 Abs. 4 MAR an keiner Stelle von einer Abwägung des Emittenten- mit dem Marktinteresse spricht; vgl. ESMA, Final Report Guidelines on the Market Abuse Regulation - market soundings and delay of disclosure of inside information, 13 July 2016, ESMA/2016/1130 Rdn. $42 \mathrm{ff}$.

90 Vgl. bereits KLÖHN, ZHR 178 (2014), 55, 86f, 89. 


\section{Lösung der Beispielsfälle}

Nach alledem sind die eingangs erwähnten Beispielsfälle wie folgt zu lösen: Entscheidet in Beispiel 2 der Vorstand über den Aufschub der Ad-hoc-Publizität, der auch zum Zeitpunkt der Gesetzesverletzungen im Amt war, so kommt es zunächst darauf an, ob konkrete Anzeichen dafür bestehen, dass dieser Vorstand gesellschaftsrechtliche Verantwortung für dieses Fehlverhalten nachgeordneter Mitarbeiter trägt. Ist die Frage zu verneinen, genießt der Vorstand bei der Entscheidung darüber, ob der Aufschub der Ad-hoc-Publizität im Emittenteninteresse i.S.v. Art. 17 Abs. 4 MAR liegt, einen Beurteilungsspielraum. Das Gericht prüft also nicht vollumfänglich, ob die Aktionäre von dem Aufschub einen höheren Nutzen als Kosten hatten. Ausreichend ist, dass der Vorstand plausibel begründet, weshalb die Kosten-Nutzen-Rechnung zugunsten des Aufschubs ausfällt. Dies kann ihm insbesondere dann gelingen, wenn er darlegt, dass die konkrete Gefahr einer Überreaktion des Marktes bestand oder dass er ohne schuldhaftes Zögern konkrete Schritte zur Verbesserung der Compliance ergriffen hat, die durch die sofortige Ad-hoc-Publizität erschwert worden wären.

Anders liegen die Dinge, wenn konkrete Tatsachen den Verdacht rechtfertigen, dass der Vorstand nicht unbefangen über den Aufschub der Ad-hoc-Publizität entscheidet, vor allem weil zu befürchten ist, dass er seine eigene gesellschaftsrechtliche Verantwortung für die Gesetzesverletzungen vertuschen möchte. In diesem Fall unterliegt die Kosten-Nutzen-Rechnung einer uneingeschränkten gerichtlichen Nachprüfung. Sie dürfte angesichts der Anzeichen für eine Verstrickung des Vorstands grundsätzlich zugunsten der sofortigen Ad-hoc-Publizität ausfallen. ${ }^{91}$ Der Aufschub der Ad-hoc-Publizität scheitert also bereits an der Voraussetzung des „Emittenteninteresses“, d.h. an der Corporate-Governance-Schranke des Art. 17 Abs. 4 MAR. Diese Konsequenz kann vermieden werden, wenn Vorstand und/oder Aufsichtsrat dafür sorgen, dass nur unbefangene Mitglieder über den Aufschub der Ad-hoc-Publizität entscheiden.

In Beispiel 1 sind keine Gesetze verletzt worden. Auch Pflichtverletzungen, etwa seitens des Produktvorstands, sind nicht unmittelbar ersichtlich. Eine konkrete Gefahr, dass der Vorstand seine Aufschubentscheidung nach Art. 17 Abs. 4 MAR nicht allein im Interesse der Aktionäre treffen wird, besteht auch aus anderen Gründen nicht. Der Vorstand des Elektronikgeräteherstellers $E$ ist damit unbefangen. Ihm steht für die Beurteilung des Emittenteninteresses i.S.d. Art. 17 Abs. 4 MAR ein Spielraum zu. Das Gericht kann die Aufschubentscheidung nur einer Plausibilitätskontrolle unterziehen. Für eine Anerkennung des Emittenteninteresses an einem Aufschub hat der Vorstand seine Entscheidung mithin $\mathrm{zu}$ plausibilisieren, indem er die Tatsachenbasis seiner 
Entscheidung substantiiert und die daran anknüpfenden Schlussfolgerungen nachvollziehbar und hinreichend ausführlich darlegt. ${ }^{92}$ Hierfür kommt es dann auf die näheren Umstände an, wie sie in den hier dargebotenen Abwandlungen des Beispiels 1 bereits aufscheinen: Lässt sich das Qualitätsproblem beheben und beabsichtigt der Vorstand die Aufschubperiode hierfür zu nutzen ${ }^{93}$ oder handelt es sich bei den Qualitätserwartungen des Marktes an die Kühlschränke und sonstigen Produkte des $E$ um eine unrealistische Übersteigerung ${ }^{94}$ ? Besteht die Gefahr einer Marktüberreaktion, welche etwa durch Marketingmaßnahmen und Informationskampagnen während der Aufschubperiode eingedämmt werden kann $?{ }^{95}$ Oder ist zu erwarten, dass der zeitweilige Aufschub als „Vertuschung“ gewertet und daher vom Markt besonders hart bestraft würde? ${ }^{96}$ Beantwortet der Vorstand diese Fragen aus der maßgeblichen Ex-anteSicht ${ }^{97}$ im Sinne eines bestehenden Aufschubinteresses des Emittenten und kann er diese Antworten mit tatsachengestützten Argumenten plausibilisieren, hat das erkennende Gericht seine Bejahung des Emittenteninteresses an einem Aufschub zu akzeptieren. Dies gilt gerade auch dann, wenn sich im Nachhinein herausstellen sollte, dass etwa die Beseitigung des Qualitätsproblems schwieriger ist als gedacht oder Marketing- und Informationskampagnen nicht die gewünschte Wirkung entfalten.

\section{Thesen}

1. Die Unternehmensreputation gehört zu den wichtigsten Vermögensgegenständen eines Emittenten. Reputationsschädliche Informationen können daher kursrelevant und bei Vorliegen der weiteren Voraussetzungen gem. Art. 17 Abs. 1 MAR ad-hoc-pflichtig sein. Möchte der Emittent die Veröffentlichung aufschieben, kann er dies nur nach Maßgabe des Art. 17 Abs. 4 MAR tun. Hiernach muss der Aufschub namentlich im Emittenteninteresse liegen.

2. Der Begriff des Emittenteninteresses in Art. 17 Abs. 4 MAR meint das Interesse der Aktionäre des Emittenten in ihrer Gesamtheit. Dieses Interesse ist auf die Maximierung des Fundamentalwerts der Gesellschaft, also des Emittenten, gerichtet. Der Aufschub ist im so verstandenen Emittenteninteresse, wenn der Erwartungsnutzen des Aufschubs für die Aktionäre höher ist als die erwarteten Kosten des Aufschubs.

92 S.o. III.3.

93 S.o. III.2.c)aa).

94 Vgl. o. III.2.d)bb).

95 S.o. III.2.c)bb).

96 S.o. III.2.d)aa).

97 S.o. III.3. 
3. Bei reputationsschädigenden Insiderinformationen führt das insofern anzustellende Kosten-Nutzen-Kalkül zu einer häufig schwierigen Abwägung: Einerseits wollen die Aktionäre, dass das Reputationskapital möglichst erhalten bleibt. Andererseits wollen sie nicht auf die Disziplinierungswirkung der Ad-hoc-Publizität für Vorstand und Aufsichtsrat verzichten. Je höher aber der Ex-post-Schutz des Reputationskapitals, desto niedriger die Ex-ante-Disziplinierungswirkung der Ad-hoc-Publizität.

a) Die Kosten der unverzüglichen (und nicht erst späteren) Ad-hoc-Publizität reputationsschädlicher Information liegen in dem Verzicht auf die zu erwartende Minderung des Reputationsvermögensschadens durch Maßnahmen während der Aufschubperiode, die durch die Ad-hoc-Publizität vereitelt oder erschwert würden. Solche Kosten können sich zudem dann ergeben, wenn eine Überreaktion des Marktes auf die unverzügliche Offenlegung hin zu befürchten steht, welcher durch Maßnahmen während der Aufschubperiode entgegengewirkt werden könnte. Keine im Rahmen des Emittenteninteresses zu berücksichtigende Kostenposition ist hingegen der Verlust von Überrenditen, welche durch die Aufrechterhaltung des (tatsächlich unberechtigt) unbefleckten Rufs während der Aufschubperiode erzielt werden können.

b) Die unverzügliche Ad-hoc-Publizität reputationsschädigender Information kann für die Aktionäre auch Nutzen stiften. Der Aufschub hat nicht nur das Potenzial zur Senkung des Reputationsschadens, sondern kann diesen auch vertiefen, wenn etwa der Markt die fortgesetzte Geheimhaltung als „Vertuschung" wahrnehmen würde. Darüber hinaus kann die unverzügliche Offenlegung zur Senkung von Agenturkosten beitragen, und zwar zunächst ganz allgemein durch Korrektur des überbewerteten Aktienkurses. Betrifft die reputationsschädliche Information Missmanagement und Compliance-Probleme des Emittenten, wirkt die drohende Marktsanktion bei unverzüglicher Offenlegung ex ante als Mittel der Disziplinierung des Vorstands und senkt auf diese Weise ebenfalls die Agenturkosten der Aktionäre.

4. Es entspricht im Grundsatz dem Interesse der Aktionäre, dass diese schwierige Abwägungsentscheidung von dem hierzu befähigteren Vorstand getroffen wird und nicht von den Gerichten. Daher steht dem Vorstand bei der Bestimmung des Emittenteninteresses i.S.d. Art. 17 Abs. 4 MAR - gerade auch im Interesse der Aktionäre in ihrer Gesamtheit - grundsätzlich ein gerichtlich nicht kontrollierbarer Beurteilungsspielraum zu. Dem Emittenten obliegen jedoch Begründungs-, Substantiierungs- und Dokumentationslasten, die sich für den handelnden Vorstand zu (Organ-)Pflichten erhärten. Im konkreten Zugriff bedarf es namentlich eines Hinweises auf konkrete Strategien zur Behebung von Reputationsschadensursachen während der Aufschubperiode und der Plausibilisierung ihrer Erfolgschancen ex ante.

5. Der Beurteilungsspielraum des Vorstands bei der Bestimmung des Emittenteninteresses steht unter dem Vorbehalt, dass der Vorstand über den Aufschub 
unbefangen entscheiden kann, hierbei also nicht durch ein Sonderinteresse beeinträchtigt wird. Ein Beurteilungsspielraum scheidet also aus, wenn konkrete Umstände - und nicht bloß abstrakte Befürchtungen - vorliegen, die besorgen lassen, dass der Vorstand die Entscheidung über den Aufschub nicht allein im Interesse der Aktionäre treffen wird. Dies bestimmt sich aus einer objektiven Ex-ante-Perspektive zum Zeitpunkt der Aufschubentscheidung.

6. Ist der Vorstand befangen, hängen die Rechtsfolgen von seinem weiteren Verhalten ab: Soll der grundsätzlich bestehende Beurteilungsspielraum für die Ermittlung des Emittenteninteresses erhalten bleiben, dürfen die befangenen Organwalter an der Aufschubentscheidung nicht teilnehmen. Tun sie es doch, entfällt der Beurteilungsspielraum. Die Frage, ob der Aufschub im Interesse des Emittenten ist, unterliegt dann der vollen Überprüfung durch die Gerichte. 